\title{
The Uses and Abuses of the Acoustic Analogy in Helicopter Rotor Noise Prediction
}

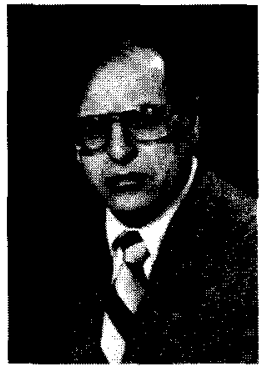

F. Farassat

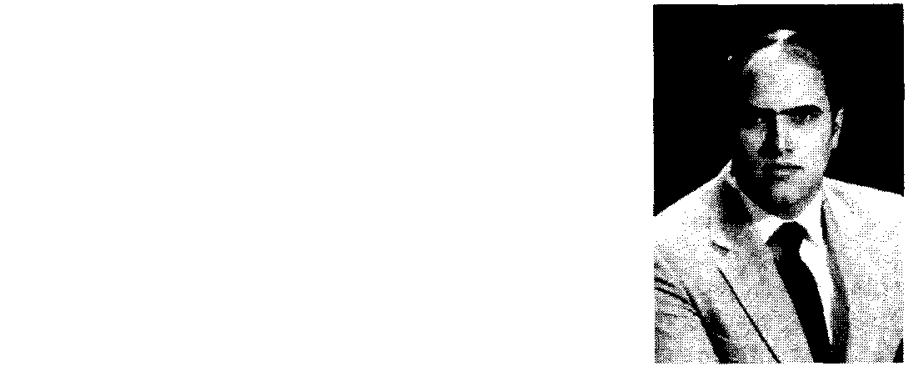

Kenneth S. Brentner

Research Engineer

NASA Langley Research Center, Hampton, Va.

\begin{abstract}
This paper is theoretical in nature and addresses applications of the acoustic analogy in helicopter rotor noise prediction. It is argued that in many instances the acoustic analogy has not been used with care in rotor noise studies. By this it is meant that approximate or inappropriate formulations have been used. By considering various mechanisms of noise generation, such abuses are identified and the remedy is suggested. The mechanisms discussed are thickness, loading, quadrupole, and blade-vortex interaction noise. The quadrupole term of the Ffowcs Williams-Hawkings equation is written in a new form which separates the contributions of regions of high gradients such as shock surfaces. It is shown by order of magnitude studies that such regions are capable of producing noise with the same directivity as the thickness noise. The inclusion of this part of quadrupole sources in current acoustic codes is quite practical. Some of the difficulties with the use of loading noise formulations of the first author in predictions of blade-vortex interaction noise are discussed. It appears that there is a need for development of new theoretical results based on the acoustic analogy in this area. Because of the impulsive character of the blade surface pressure, a time scale of integration different from that used in loading and thickness computations must be used in a computer code for prediction of blade-vortex interaction noise.
\end{abstract}

\section{Introduction} $\mathrm{n}$ the last decade, there has been an intense interest in helicopter noise prediction. Passenger comfort, impact on communities under the flight path, and detectability are some of the reasons for this interest. Helicopter manufacturers realize that in the future the noise issue will be an important factor in the domestic and the international markets. As is well known, the prediction of helicopter noise is a very difficult problem. The theoretician has much to contribute to the solution of this problem. One of the most successful methods of attacking the problem is through the use of the acoustic analogy of Lighthill, Ref. 1. The fundamental equation relevant to rotor noise prediction was published in 1969 by Ffowcs Williams and Hawkings, Ref. 2, and is now known by their names as the Ffowcs Williams-Hawkings (FW-H) equation.

This paper is theoretical in nature with the following aims: (i) to describe the uses and the power of the acoustic analogy in rotor noise prediction; and (ii) to describe some of the abuses of the acoustic analogy and how they can be corrected. The authors do not claim that the abuses in the past have been intentional but that they could have been avoided with some care in the application of the acoustic analogy.

Presented at the AHS Specialists' Meeting on Aerodynamics and Aeroacoustics, Arlington, Texas, February 25-27, 1987.
One of the mathematical subtleties associated with the FW$H$ equation is the use of generalized functions and advanced differential geometry. Those who have tried to derive acoustic formulations for rotor noise calculation have discovered that there are many equivalent results, each having its own merits and shortcomings for number crunching, Ref. 3. It is also true that in deriving these results, some algebraic manipulations become extremely complicated and intractable. The use of advanced mathematics, such as generalized function theory and differential geometry, becomes imperative to simplify the derivation of the acoustic formulation and to give geometrical meaning to the terms appearing in the result. We will present several convincing examples of this fact. It is the opinion of the authors that in the prediction of rotor noise, mathematics plays as important a role as does fluid mechanics, experiments, or computers. It is this aspect of noise prediction which will be highlighted in the paper.

\section{The Acoustic Analogy}

Physically, the acoustic analogy replaces the effect of fluid motion in generation and propagation of sound by fictitious sources in an undisturbed fluid, Ref. 1. The strength of these sources are assumed to be known. In rotor acoustics, this means that blade surface pressure and the flow around the blade must be known. Of course, this information is very difficult to obtain 
and much more work needs to be done both experimentally and theoretically. The requirement of aerodynamic input data is viewed by some as a weakness of the acoustic analogy. We will argue here that it is not.

Mathematically, the application of the acoustic analogy is a boundary element method; as such, it is, in principle, able to supply its own input data. In the case of high speed propellers where the flow around the blades is much less complicated than helicopter rotors, the aerodynamic theory based on the acoustic results has been published by Hanson, Ref. 4, and Farassat and Myers, Ref. 5. Although this can also be accomplished for rotors, it is more appropriate to use a direct numerical approach based on the Euler or Navier-Stokes equations. Most aerodynamic codes give satisfactory results near the blades because of small grid sizes, but the accuracy deteriorates far from the blades. It is thus necessary to use the acoustic analogy when the observer is in regions far from the blades, utilizing the aerodynamic information on and around the blades.

Several methods to replace the acoustic analogy are available. Hawkings, Ref. 6, has proposed the use of numerical aerodynamic calculations to obtain boundary data on a surface surrounding the rotor and then using Kirchhoff's integral for an observer in the region exterior to the surface. Korkan, Von Lavante, and Bober, Ref. 7, have had some success with this method for high speed propellers. The method suffers from lack of resolution of aerodynamic data on the boundary surface. Kirchhoff's formula has also been used in rotor acoustics by Lyrintzis and George, Ref. 8, and Purcell, Strawn and Yu, Ref. 9, for high speed blade-vortex interaction noise studies. Recently, Farassat and Myers, Ref. 10, have derived and extended a general Kirchhoff's formula for moving surfaces by Morgans, Ref. 11. The final result is presented in a form suitable for use on a computer. Recently, a purely numerical wave propagation study has been attempted by Baeder, McCroskey, and Srinivasan, Ref. 12. This shows some promise for the future, particularly when more powerful computers become available.

Since the unsteady flow field around rotors is very complex, it is very likely that, in the next decade, the attention of aerodynamicists will be concentrated only on the prediction of the flow field around the blades. The acoustic analogy thus will remain a very useful tool in the future in rotor noise prediction. As will be shown below, many further improvements can be made in the application of the acoustic analogy which will extend its applicability and usefulness.

\section{The Governing Equation in a New Form}

In this section, we will write the FW-H equation in a new form by manipulating the quadrupole term in such a way that various noise generation mechanisms can be distinguished clearly. The FW-H equation in its conventional form is the following wave equation:

$$
\begin{aligned}
\square^{2} p^{\prime}= & \frac{\partial}{\partial t}\left[\rho_{o} v_{n}|\nabla f| \delta(f)\right] & & \text { thickness } \\
& -\frac{\partial}{\partial x_{i}}\left[\ell_{i}|\nabla f| \delta(f)\right] & & \text { loading } \\
& +\frac{\partial^{2}}{\partial x_{i} \partial x_{j}}\left[T_{i j} H(f)\right] & & \text { quadrupole }
\end{aligned}
$$

where $p^{\prime}$ is $c^{2}\left(\rho-\rho_{o}\right)$ which is essentially the acoustic pressure. Here, $\rho_{o}$ and $c$ are the density and speed of sound in the undisturbed medium, $v_{n}$ and $\ell_{i}$ are the local normal velocity and force intensity on the body, and $T_{i j}$ is the Lighthill stress tensor. The body in motion is defined by $f(\vec{x}, t)=0$, such that $f>0$ outside the body. The functions $H(f)$ and $\delta(f)$ are the Heaviside and the Dirac delta functions, respectively.
It is a useful practice to relate the various rotor noise generation mechanisms to the source term in the FW-H equation. For example, the blade-vortex interaction noise is described by the loading noise term of the FW-H equation since the noise is generated by the sudden change in the blade surface pressure. We will now write the quadrupole term in Eq. (1) in a form which is more suitable for theoretical and numerical analysis than the conventional form given above. The derivation of this result is presented in Ref. 13.

We begin by giving some definitions first. Consider the flow around an airfoil at high speed shown in Fig. 1. The body (rotor blade) is described by $f=0$. We have regions with high velocity gradients in the boundary layer and the wake and discontinuities in velocity and pressure across the shocks. The shock surfaces are defined by the equation $k=0, \tilde{k}>0$. Their edges are defined by $k=\vec{k}=0$. The unit normals to the body and shock surfaces are defined by $\vec{n}$ and $\vec{n}^{\prime}$, respectively. Let the trailing edge (TE) of the blade be specified by equations $f=\tilde{f}=0$ with the inward unit geodesic normal denoted by $\vec{v}$. The geodesic normal is tangent to the airfoil surface and normal to the TE, Fig. 2. Note that, at the TE, there are two geodesic normals corresponding to the upper and the lower surfaces meeting at the TE. The inward unit geodesic normal of the shock surface is denoted by $\vec{v}^{\prime}$.

We now define the symbols related to the Lighthill stress tensor $T_{i j}$. Let the components of vectors $\vec{Q}$ and $\vec{q}$ be defined as

$$
\begin{aligned}
Q_{i} & =T_{i j} n_{j} \\
q_{i} & =\Delta\left(T_{i j} n_{j}^{\prime}\right)
\end{aligned}
$$

where $\Delta=()_{2}-()_{1}$ and the subscripts refer to regions 1 and 2 across the shock in Fig. 1. The following symbols are also needed:

$$
\begin{aligned}
& Q_{n}=\vec{Q} \cdot \vec{n}=T_{i j} n_{i} n_{j} \\
& \vec{Q}_{r}=\vec{Q}-Q_{n} \vec{n}
\end{aligned}
$$

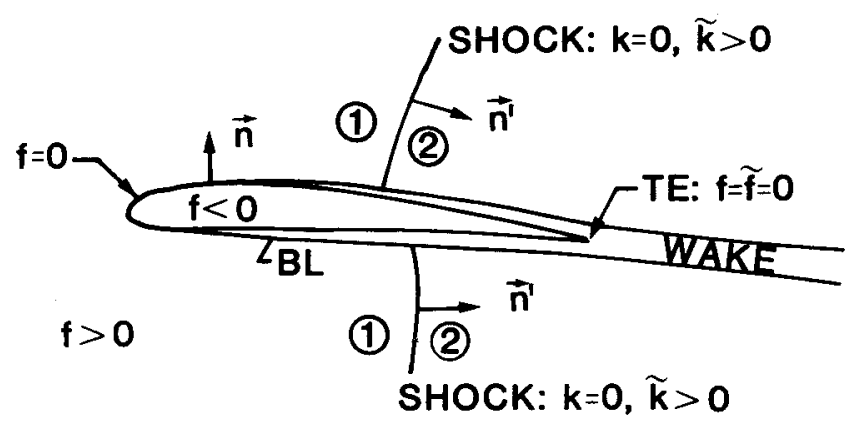

Fig. 1 Schematic of high speed flow around an airfoil.

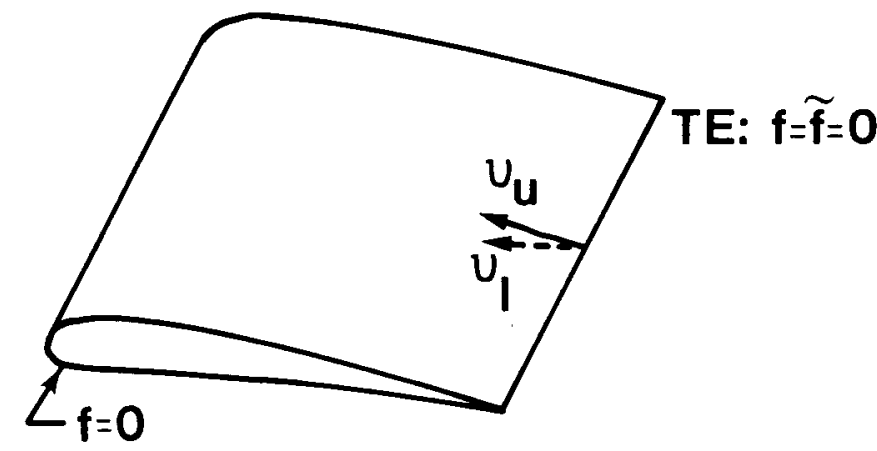

Fig. 2 The geodesic normals at the trailing edge of a blade. 


$$
\begin{aligned}
q_{n^{\prime}} & =\vec{q} \cdot \vec{n}^{\prime} \\
\vec{q}_{T} & =\vec{q}-q_{n^{\prime}} \vec{n}^{\prime} \\
Q_{v} & =\vec{Q} \cdot \vec{v} \\
& =T_{i j} v_{i} n_{j} \\
q_{v^{\prime}} & =\vec{q} \cdot \vec{v}^{\prime} \\
& =\Delta T_{i j} v_{i}^{\prime} n_{j}^{\prime} \\
Q_{n^{\prime}}^{\prime} & =T_{i j} n_{i}^{\prime} n_{j}^{\prime} \\
Q_{G} & =T_{i j} \frac{\partial n_{i}}{\partial x_{j}} \\
q_{G} & =\Delta T_{i j} \frac{\partial n_{i}^{\prime}}{\partial x_{j}}
\end{aligned}
$$

With these definitions, we can now write the quadrupole term of the FW-H equation as follows:

$$
\begin{aligned}
& \frac{\partial^{2}}{\partial x_{i} \partial x_{j}}\left[T_{i j} H(f)\right]=\frac{\partial^{2} T_{i j}}{\partial x_{i} \partial x_{j}} H(f) \quad \quad\left\{\begin{array}{c}
\text { pure } \\
\text { quadrupole } \\
\text { term }
\end{array}\right. \\
& \left.\begin{array}{l}
+\left[2 \nabla_{2} \cdot \vec{Q}_{T}+\frac{\partial Q_{n}}{\partial n}-4 H_{f} Q_{n}-Q_{G}\right] \delta(f) \\
+Q_{n} \delta^{\prime}(f)
\end{array}\right\} \begin{array}{c}
\text { blade } \\
\text { surface } \\
\text { terms }
\end{array} \\
& \left.\begin{array}{l}
+\left[2 \nabla_{2} \cdot \vec{q}_{T}+\Delta\left(\frac{\partial Q_{n^{\prime}}^{\prime}}{\partial n^{\prime}}\right)-4 H_{k} q_{n^{\prime}}-q_{G}\right] \delta(k) \\
+q_{n^{\prime}} \delta^{\prime}(k)+q_{v^{\prime}} \delta(\tilde{k}) \delta(k)
\end{array}\right\} \begin{array}{l}
\text { shock } \\
\text { surface } \\
\text { terms }
\end{array} \\
& +\left[Q_{v}\right] \delta(f) \delta(\delta f) \\
& \left\{\begin{array}{c}
\text { trailing } \\
\text { edge } \\
\text { term }
\end{array}\right.
\end{aligned}
$$

In this equation, $H_{f}$ and $H_{k}$ are the local mean curvature of the body and the shock surface, respectively. Also, the square bracket in the trailing edge term stands for $\left(Q_{v}\right)_{\text {upper }}+\left(Q_{v}\right)_{\text {lower }}$ where the subscripts refer to the upper and lower surfaces meeting at the trailing edge. The surface divergence operator, Ref. 14, is denoted by $\nabla_{2}$. The quadrupole term in the FW-H, Eq. (1) can therefore be broken down into components which clearly show the contribution of various noise sources. The application of Eq. (4) will be discussed below. Some of the terms in this equation can be simplified further. For example, the last term in the shock surface terms will be shown by shock jump relations to vanish. Note that, in the original version of this paper, there were two errors in Eq. (4) (term $Q_{G}$ and $q_{G}$ missing), which are corrected here.

\section{The Uses, Abuses, and Their Remedy}

We will now concentrate on the applications of the solution of the FW-H equation. We consider three areas of use in rotor acoustics: i) thickness and loading noise, ii) quadrupole noise, and iii) blade-vortex interaction noise. In each case, we point out the incorrect use or "the abuse" and then describe the remedy.

\section{i) Thickness and Loading Noise}

One of the simplest formulations for prediction of thickness and loading noise was given by Farassat, Ref. 15, as follows:

$$
\begin{aligned}
4 \pi p^{\prime}(\vec{x}, t)= & \frac{1}{c} \frac{\partial}{\partial t} \int_{F=0}\left[\frac{p_{o} v_{n}+p \cos \theta}{r \Lambda}\right]_{r e t} d \Sigma \\
& +\int_{F=0}\left[\frac{p \cos \theta}{r^{2} \Lambda}\right]_{r e t} d \Sigma
\end{aligned}
$$

Here, we assume $\ell_{i}=p n_{i}$ ( $p \equiv$ blade surface pressure), $F=$ $[f(\vec{y}, \tau)]_{\text {ret }}=f(\vec{y}, t-r / c)$ and $\theta$ is the angle between the normal to the body $\vec{n}$ and the radiation direction $\vec{r}=\vec{x}-$ $\vec{y}$. The source and observer space-time variables are denoted by $(\vec{y}, \tau)$ and $(\vec{x}, t)$, respectively. The area of the surface $F=$ 0 is denoted by $\Sigma$. The symbol $\Lambda$ stands for

$$
\Lambda=\left(1+M_{n}^{2}-2 M_{n} \cos \theta\right)^{1 / 2}
$$

where $M_{n}=v_{n} / c$ is the local normal Mach number. Equation (5) is used for both steady and unsteady loading noise. Here, we consider nonimpulsive surface pressure only. The observer time derivative in Eq. (5) is taken numerically.

In the application of Eq. (5), the following relations are used in place of $\frac{d \Sigma}{\Lambda}$ since direct construction of the $\Sigma$-surface is difficult, Ref. 15:

$$
\begin{aligned}
\frac{d \Sigma}{\Lambda} & =\frac{d S}{\left|1-M_{r}\right|} \\
& =\frac{c d \tau d \Gamma}{\sin \theta}
\end{aligned}
$$

where $d S$ is element of the surface area of the body $f=0$, and $M_{r}$ is the local Mach number of the body in the radiation direction. The symbol $d \Gamma$ is the element of the arc length along the intersection of the sphere $r=c(t-\tau)$ with the body $f=$ 0 . It was thought originally that, since there is a singularity at $M_{r}=1$ in Eq. (7-a), use of Eq. (7-b) in Eq. (5) would give a result valid in all range of Mach numbers, Refs. 16 and 17. This result is:

$$
\begin{aligned}
4 \pi p^{\prime}(\vec{x}, t)= & \frac{\partial}{\partial t} \int \frac{\rho_{0} v_{n}+p \cos \theta}{r \sin \theta} d \Gamma d \tau \\
& +\int \frac{c p \cot \theta}{r^{2}} d \Gamma d \tau
\end{aligned}
$$

It was found, however, that this equation is suitable for high speed blade motion but the accuracy deteriorated substantially at low speed. The reason is that, at low speed, the line integral in Eq. (8) changes rapidly so that the time integration must be performed at a much finer scale, which is impractical due to the excessive computer time usage. Therefore, Eq. (7-a) was used for $\frac{d \Sigma}{\Lambda}$ in Eq. (5) for low speed. Such switching of the formulation was performed automatically in Ref. 17 with some success.

There are two basic problems with Eq. (5). First, the integral in the first term on the right must be evaluated twice at $t$ and $t+d t$ for numerical differentiation. This increases computation time. The second problem is that no matter how accurately the integration is performed, the numerical differentiation amplifies the errors, which leads to unrealistic high frequency oscillations in acoustic signatures, Ref. 17. Equation (5) has 
also some problems for impulsive surface pressure, which will be discussed later. The remedy for the above problems was to take the time derivative in $\mathrm{Eq}$. (5) inside the first integral. Two distinct formulations for low and high speeds were derived and used with much success, Refs. 18 and 19. Brentner gives some applications of the subsonic formulation to helicopter rotors in Ref. 20.

\section{ii) The Quadrupole Noise}

There has been much interest in the quadrupole noise prediction of rotors following the pioneering work of Hanson and Fink, Ref. 21, and the work for helicopter rotors by Schmitz and $\mathrm{Yu}, \mathrm{Ref} .22$. It is not difficult to write the solution to the quadrupole term of the FW-H equation:

$$
4 \pi p_{Q}^{\prime}(\vec{x}, t)=\frac{\partial^{2}}{\partial x_{i} \partial x_{j}} \int_{F>0} \frac{T_{i j}}{r} c d \Omega d \tau
$$

where $p_{Q}^{\prime}$ is the acoustic pressure due to quadrupoles and $d \Omega$ is element of the surface area of the sphere $r=c(t-\tau)$. The integration is carried out in the exterior region of the surface $F=[f(\vec{y}, \tau)]_{\text {ret }}=0$. Assuming that the flow around the blades is known, use of Eq. (9) as it stands is a monumental task if the differentiation with respect to the space variables is performed numerically. In addition, a volume integration must be carried out over a suitably large volume.

A way around numerical differentiation with respect to the space variables of Eq. (9) is to convert these variables to the observer time variable, as was done for loading noise, Ref. 15. A simple, but approximate, method to accomplish this is to use the far field approximation

$$
\frac{\partial^{2}}{\partial x_{i} \partial x_{j}} \simeq \frac{\hat{r}_{i} \hat{r}_{j} \partial^{2}}{c^{2}} \frac{\partial^{2}}{\partial t^{2}}
$$

where $\hat{r}_{i}=\left(x_{i}-y_{i}\right) / r$ is the unit radiation vector. This changes Eq. (9) into

$$
4 \pi p_{Q}^{\prime}(\vec{x}, t) \simeq \frac{1 \partial^{2}}{c \partial t^{2}} \int_{F>0} \frac{T_{r r}}{r} d \Omega d r
$$

where $T_{r r}=T_{i j} \hat{r}_{i} \hat{r}_{j}$. Equation (11) is only valid in the far field. This, however, is not entirely satisfactory since, in some of the applications of Eq. (11), one may want to predict the quadrupole noise in the near and intermediate fields. A more compelling reason to have an exact result equivalent to Eq. (9) will be given below.

Equation (9) can in fact be written as

$$
\begin{aligned}
4 \pi p_{Q}^{\prime}(\vec{x}, t) & =\frac{\partial^{2}}{\partial x_{i} \partial x_{j}} \int \frac{T_{i j}}{r} \delta(g) H(f) d \vec{y} d \tau \\
& =\int H(f) T_{i j} \frac{\partial^{2}}{\partial x_{i} \partial x_{j}}\left[\frac{\delta(g)}{r}\right] d \vec{y} d \tau
\end{aligned}
$$

where $g=r-t+\frac{r}{c}$. It can be shown that

$$
\begin{aligned}
\frac{\partial^{2}}{\partial x_{i} \partial x_{j}}\left[\frac{\delta(g)}{r}\right]= & \frac{1}{c^{2}} \frac{\partial^{2}}{\partial t^{2}}\left[\frac{\hat{r}_{i} \hat{r}_{j} \delta(g)}{r}\right] \\
& +\frac{1}{c} \frac{\partial}{\partial t}\left[\frac{\left(3 \hat{r}_{i} \hat{r}_{j}-\delta_{i j}\right) \delta(g)}{r^{2}}\right]
\end{aligned}
$$

$$
+\frac{3 \hat{r}_{i} \hat{r}_{j}-\delta_{i j}}{r^{3}} \delta(g)
$$

where $\delta_{i j}$ is the Kronecker delta. Using this equation in Eq. (12), we obtain

$$
\begin{aligned}
4 \pi p_{Q}^{\prime}(\vec{x}, t)= & \frac{1}{c \partial t^{2}} \int_{F>0} \frac{T_{r r}}{r} d \Omega d \tau \\
& +\frac{\partial}{\partial t} \int_{F>0} \frac{3 T_{r r}-T_{i i}}{r^{2}} d \Omega d \tau \\
& +c \int_{F>0} \frac{3 T_{r r}-T_{i i}}{r^{3}} d \Omega d \tau
\end{aligned}
$$

Here the summation convention over the index $i$ is used. This relation is now exactly equivalent to Eq. (9). It is obvious that, up to about one radius from the blade tip, the last two terms are of the same order as the first term. Because of the nature of the integrals in Eq. (14), which are usually strong functions of observer time, it is estimated that the second integral involving a time derivative should at least be included in the intermediate (between near and far) field.

There is one area where Eq. (14) is superior to Eq. (11) in application. Because of complexity of the rotor acoustic codes, these codes should be checked very carefully. Since Eq. (14) is in principle exact, moving the observer to the vicinity of the rotor can be used together with Eq. (5) to test if the aerodynamic input data can be recovered from the acoustic code. Equation (11) does not allow this important check of the acoustic code.

Even Eq. (14) demands too much from the aerodynamicist. Does one really need to know everything about the quadrupoles in the near field? There are regions of large velocity gradients around airfoils moving at high speed. These regions are as follows:

a) The boundary layer

b) The shock surfaces

c) Tip vorticity and the wake

None of the current quadrupole theories bring out the contributions of these regions clearly. On intuitive grounds, it can be argued that the contribution of these regions to $p_{Q}^{\prime}$ should be substantial. We observe that Eq. (4) indeed can be used to study this problem, which will be presented next. First, however, we note that the pure quadrupole term of Eq. (4) includes part of the boundary layer and all of the tip vorticity and wake contributions.

\section{a. The Boundary Layer}

The boundary layer contribution consists of four terms in Eq. (4) as shown below:

$$
\begin{aligned}
E_{B L}= & {\left[\frac{\partial^{2} T_{i j}}{\partial x_{i} \partial x_{j}}\right]_{B L} H(f) } \\
& +\left[2 \nabla_{2} \cdot \vec{Q}_{T}+\frac{\partial Q_{n}}{\partial n}-4 H_{f} Q_{n}-Q_{G}\right] \delta(f) \\
& +Q_{n} \delta^{\prime}(f)+\left[Q_{v}\right] \delta(\tilde{f}) \delta(f)
\end{aligned}
$$

The first term gives a volume integral, the second and third terms give surface integrals, and the last term gives a line integral over the trailing edge. We will next study the contribution of the volume term. The boundary layer contribution to $p_{Q}^{\prime}$, denoted by $p_{Q B L}^{\prime}$, can be written as follows: 


$$
4 \pi p_{Q B L}^{\prime}(\vec{x}, t)=\int \frac{E_{B L}}{r} \delta(g) d \vec{y} d \tau
$$

where $g=\tau-t+r / c$.

The volume term of Eq. (15) in Eq. (16) can be written in a readily calculable form:

$$
\begin{aligned}
& \int \frac{H(f)}{r}\left[\frac{\partial^{2} T_{i j}}{\partial y_{i} \partial y_{j}}\right]_{B L} \delta(g) d \vec{y} d \tau= \\
& \iint_{\tau} \frac{c}{r \sin \theta}\left\{\int_{0}^{h}\left[\frac{\partial^{2} T_{i j}}{\partial y_{i} \partial y_{i}}\right]_{B L} d h\right\} d \Gamma d \tau
\end{aligned}
$$

where the interior integral is a line integral over the width of the boundary layer on the sphere $r=c(t-\tau)$. Referring to Fig. 3, it is seen that the main contribution of the integrand is due to the gradient of the tangential component of velocity in the normal direction, i.e.:

$$
\begin{gathered}
{\left[\frac{\partial^{2} T_{i j}}{\partial y_{i} \partial y_{j}}\right]_{B L} \sim 2 \rho \frac{\partial u}{\partial n} \frac{\partial v}{\partial s}} \\
\int_{0}^{h}\left[\frac{\partial^{2} T_{i j}}{\partial y_{j} \partial y_{j}}\right]_{B L} d h \sim 2\left(\rho u \frac{\partial v}{\partial s}\right)^{*}
\end{gathered}
$$

where $*$ indicates some mean quantity in the boundary layer. Here $(u, v)$ are the fluid velocity components in the tangent and normal directions to the body surface with respect to the frame fixed to the undisturbed medium. This means that $u$ is of the order of blade velocity. It is seen that, because of the high gradient of velocity in the boundary layer, the integral in Eq. (18-b) may be large. Incidentally, the nature of the integrals resulting from Eq. (15) is similar to the thickness noise and gives the same directivity. It is therefore important to include these terms in rotor acoustic calculations, particularly because the volume integral in Eq. (17) can be calculated in a similar manner to the surface integrals of loading and thickness noise.

Assuming that the no-slip condition is satisfied, the last three terms of $E_{B L}$, with the exception of $\frac{\partial Q}{\partial n}$ of Eq. (15), are mainly kinematic in the sense that they are obtained from the knowledge of blade motion and geometry only. They can be written in the form of surface and line integrals by the method described in Ref. 19. Current codes can be modified to incorporate these terms. Preliminary calculations have shown a contribution of up to 15 percent of the negative peak due to the thickness noise

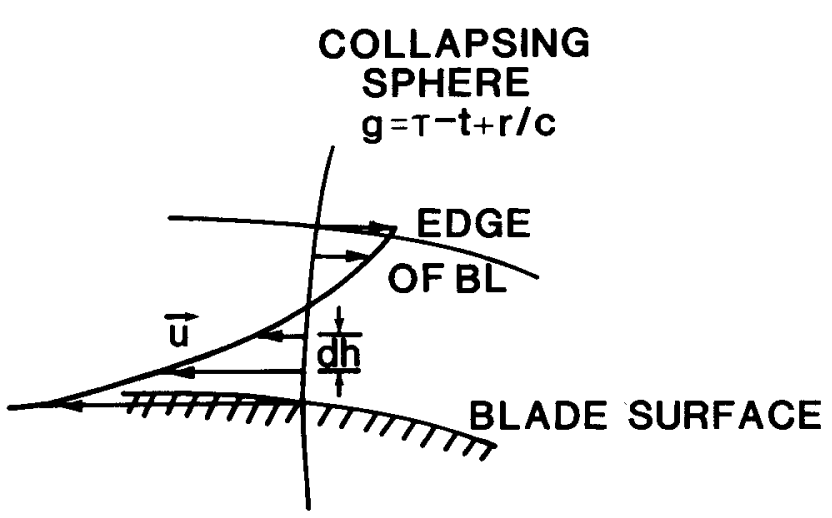

Fig. 3 Evaluation of boundary layer contribution to quadrupole noise. by the surface integrals only. Our experience with high speed propellers, Ref. 19, indicates that the line integral of the type of the trailing edge term in Eq. (15) can also be significant.

In summary, the boundary layer quadrupole contribution can be written in such a way that the current helicopter acoustic codes can be modified to evaluate it. Preliminary order-ofmagnitude analysis shows thickness-like behavior with the possibility of significant conribution to the quadrupole noise.

\section{b. The Shock Surfaces}

The contribution of the shock surfaces to $p_{O}^{\prime}$ denoted by $p_{Q S S}^{\prime}$ is described by the terms in Eq. (4) involving $\delta(k)$. Essentially, there is an infinite gradient of velocity across the shock surfaces. For this reason, it can be argued on a mathematical basis that the shock surface contribution to quadrupole noise can be important. An explicit formulation of shock surface noise was derived by Farassat, Ref. 23, and applied with limited success by Kitaplioglu and George, Ref. 24. The main difficulties were the lack of geometric interpretation of the many terms appearing in the formula as well as its complexity. The approach based on terms of Eq. (4) is much simpler.

We denote by $E_{S S}$ the shock surface source terms:

$$
\begin{aligned}
E_{S S}= & {\left[2 \nabla_{2} \cdot \vec{q}_{T}+\Delta\left(\frac{\partial Q_{n^{\prime}}^{\prime}}{\partial n^{\prime}}\right)-4 H_{f} q_{n^{\prime}}-q_{G}\right] \delta(k) } \\
& +q_{n^{\prime}} \delta^{\prime}(k)+q_{v^{\prime}} \delta(\tilde{k}) \delta(k)
\end{aligned}
$$

The two terms involving $\delta(k)$ and $\delta^{\prime}(k)$ give surface integrals over the shock and the last involving $\delta(\bar{k}) \delta(k)$ gives a line integral over the edge of the shocks. Using the unsteady shock jump relations, we can show that

$$
\begin{aligned}
& \vec{q}_{T}=\left(v_{n^{\prime}}, \Delta \rho\right) \vec{u}_{T} \\
& q_{n^{\prime}}=\left(v_{n^{\prime}}^{2}-c_{*}^{2}\right) \Delta \rho \\
& q_{v^{\prime}}=0 .
\end{aligned}
$$

Here $v_{n^{\prime}}$ is the shock speed with respect to the frame fixed to the undisturbed medium, and $\vec{u}_{T}$ is the velocity of the fluid tangent to the shock surface in this frame. The critical velocity of sound is denoted $c_{*}$. Equation (20-c) indicates that there is no line integral over the perimeter of the shock surfaces. For conventional airfoils used in helicopter rotors, the shock surfaces are normal with small curvature. This means that $\nabla_{2}$. $\vec{q}_{T}, \Delta\left(\frac{\partial Q_{n^{\prime}}^{\prime}}{\partial n^{\prime}}\right), H_{f} q_{n^{\prime}}$ and $q_{G}$ are all small, so that the term involving $\delta(k)$ is also small. One is therefore left with the following approximation of $E_{S S}$ :

$$
E_{S S} \simeq\left(v_{n^{\prime}}^{2}-c_{*}^{2}\right) \Delta \rho \delta^{\prime}(k)
$$

We will give an order of magnitude analysis of this term below. From the above equation, it is obvious that the shock contribution is dipole-like in character with the dipole axis in the direction of the motion of the blade (i.e., the peak directivity is in the rotor plane). Thus, the shock surface noise will also behave like the thickness noise.

To study the strength of the source term in Eq. (21), we assume that the blade is moving at $M=0.90$ with the flow Mach number ahead of the shock at $M_{1}=1.3$. Then we can estimate the source term as follows:

$$
\begin{gathered}
\left(v_{n^{\prime}}^{2}-c_{*}^{2}\right) \Delta \rho=c^{2}\left(M_{n^{\prime}}^{2}-0.968\right) \Delta \rho \\
M_{n^{\prime}} \sim M=0.90
\end{gathered}
$$




$$
\begin{aligned}
& \Delta \rho \sim \frac{5\left(M_{1}^{2}-1\right)}{\left(M_{1}^{2}+5\right)} \rho_{1} \simeq 0.443 \mathrm{~kg} / \mathrm{m}^{3} \\
& c^{2}\left(M_{n^{\prime}}^{2}-0.968\right) \Delta \rho \sim-8,091 . \mathrm{Pa}
\end{aligned}
$$

The source strength is therefore large enough that it should be included in quadrupole calculations.

Incidentally, the contribution of Eq. (21) to $p_{Q}^{\prime}$ can be found by the method presented in Ref. 15 as follows:

$$
\begin{aligned}
4 \pi p_{Q S S}^{\prime}(\vec{x}, t)= & \int_{K=0} \frac{1}{r} \tilde{Q}_{1}\left(E_{S S}, \dot{E}_{S S}\right) d \Sigma \\
& +\int_{K=0} \frac{1}{r^{2}} \tilde{Q}_{2}\left(E_{S S}\right) d \Sigma
\end{aligned}
$$

where $\tilde{Q}_{1}\left(E_{S S}, \dot{E}_{S S}\right)$ and $\tilde{Q}_{2}\left(E_{S S}\right)$ are some functions of source strength and $K=[k(\vec{y}, r)]_{r e t}$. Here $\dot{E}_{S S}$ is the time rate of variation of $E_{S S}$. Thus, the shock oscillation is included in Eq. (23).

It was found by Schmitz and coworkers, Ref. 25, that thickness and loading noise calculations together estimate the rotor noise with reasonable accuracy up to a tip Mach number, which essentially depends on blade thickness ratio and design. Above this Mach number the shocks in the tip region become extensive going beyond the tip region. This sudden change in flow around rotors is called delocalization by Schmitz, Ref. 15. It is the belief of the present authors that the major contribution to the quadrupole noise comes from the shock surfaces. This can be deduced from the above estimation and the fact that the surface $\Sigma: K=0$ in Eq. (23) is highly time dependent and can produce large variations in $p_{Q}$.

c. Tip Vorticity and the Wake

The tip vortex and blade wake are also regions of large velocity gradients. The source term for these in Eq. (4) is the pure quadrupole term, which is a volume term. The tip vorticity effect can be converted into a line integral along the vortex line involving the vortex strength. This can be included in current rotor acoustic codes.

The blade wake contribution can be written in such a way that only the gradient of velocity normal to the wake appears. Extensive volume integration can be avoided by confining the integration to the regions of high velocity gradients near the trailing edge of the rotor blades. Like the boundary layer noise discussed above, these calculations can also be performed as surface integration because of the small thickness of the wake. Therefore, the wake calculation can also be included in the current rotor acoustic codes.

\section{iii) Blade-Vortex Interaction Noise}

Blade-vortex interaction noise is impulsive in nature and appears as sharp pulses in the acoustic signature. The main source term for this mechanism is the loading term in the FW$\mathrm{H}$ equation, Eq. (1). Nakamura, Ref. 26, has used a result of Farassat, Ref. 15, for calculation of the vortex interaction noise. It is in the form of Eq. (5) of this paper without the thickness term. Although the surface pressure data has not been available in great detail, one can show that the disagreement with measured data thus far is mainly due to the acoustic approach. We will demonstrate this by a simple example.

Measured surface pressure data due to blade-vortex interaction shows the impulsive nature of the pressure Ref. 26 . In the following example, we assume that the impulsive blade pressure is like a delta function in time and acts on a circular region of radius $a$ in the $x_{1} x_{2}$-plane as shown in Fig. 4 .

Let the impulse be applied at the time $t=0$. We are interested in solving

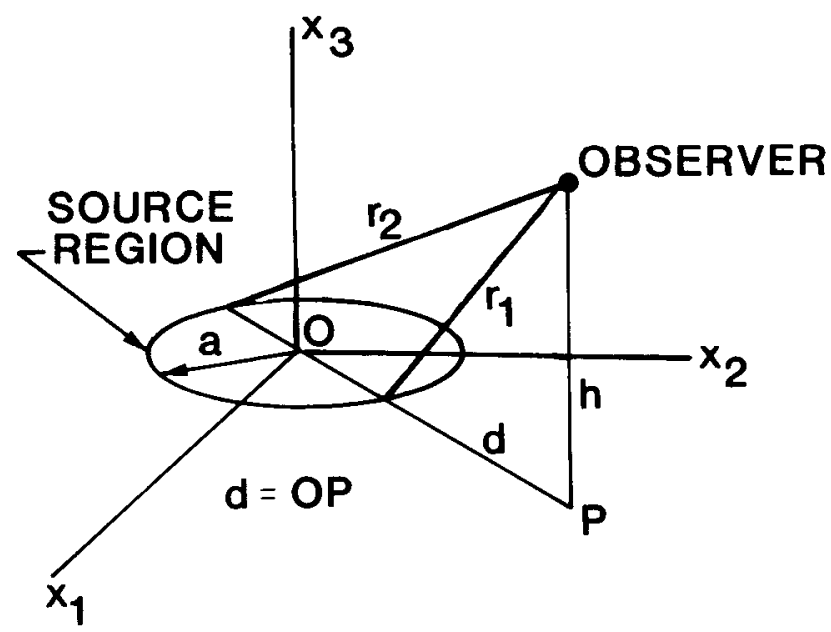

Fig. 4 The geometry of the impulsive source distribution used in the example related to blade-vortex interaction noise.

$$
\square^{2} p^{\prime}=-I\left(x_{1}, x_{2}\right) \delta(t) \delta^{\prime}\left(x_{3}\right)
$$

where $I\left(x_{1}, x_{2}\right)$ is the impulse intensity defined from

$$
I\left(x_{1}, x_{2}\right)=\int_{-\epsilon}^{\epsilon} p\left(x_{1}, x_{2}, t\right) d t
$$

Here $p$ is the surface pressure in the source region. Note that this impulsive model of blade pressure corresponds to infinite rate of change of $p$. The exact solution of Eq. (24), based on the method discussed in Refs. 3 and 15, is given as:

$$
2 \pi p^{\prime}(\vec{x}, t)=\frac{\partial}{\partial t} \int_{r=c t} \frac{l \cot \theta}{r} d \Gamma+c \int_{r=c t} \frac{I \cot \theta}{r^{2}} d \Gamma
$$

These line integrals are integrated over the intersection of sphere of radius $r=c t$ (with center at the observer) and the source region. The curve of intersection is part of a circle as shown in Fig. 5.

We will now assume that $I\left(x_{1}, x_{2}\right)=$ constant in order to show some surprising features of the acoustic field relevant to the blade-vortex interaction. Referring to Fig. 5, we can see that $\cot \theta=h / R$ where $R$ is the radius of the $\Gamma$-curve and $h$ is the distance of the observer from $x_{1} x_{2}$-plane. We distinguish two regions of space with different acoustic characteristics.

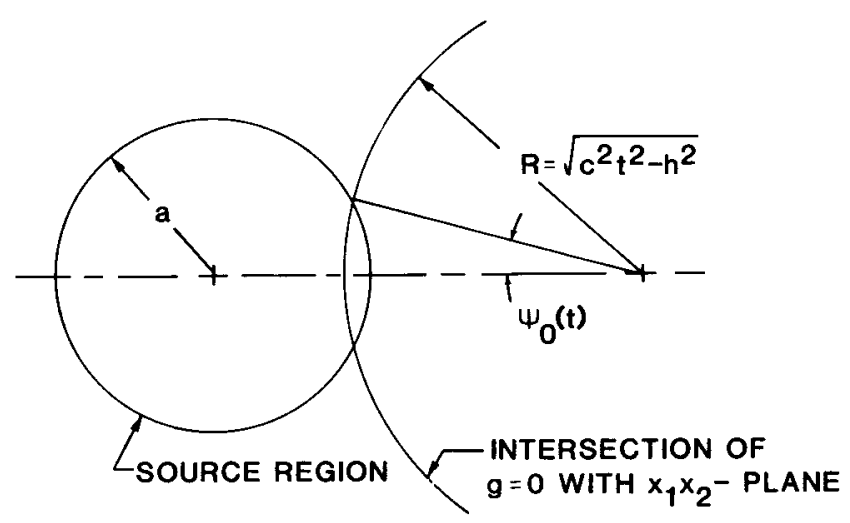

Fig. 5 The definition of angle $\psi_{o}(t)$ in the example of impulsive source distribution. 
The first is outside the cylinder of radius $a$ with axis along $x_{3}$ axis, and the second region is inside this cylinder.

If $\psi$ is the azimuthal angle along the $\Gamma$-curve, then $d \Gamma=$ $R d \psi$. For the observer in region 1 , we have

$$
\pi p^{\prime}(\vec{x}, t)= \begin{cases}0 & 0<t<\frac{r_{1}}{c} \\
\frac{h I}{c}\left\{\frac{\partial}{\partial t}\left[\frac{\psi_{0}(t)}{t}\right]+\frac{\psi_{0}(t)}{t^{2}}\right\} & \begin{array}{l}
\frac{r_{1}}{c}<t<\frac{r_{2}}{c} \\
0
\end{array} \\
t>\frac{r_{2}}{c}\end{cases}
$$

where $r_{1}$ and $r_{2}$ the distances from the observer to the nearest and farthest points of the perimeter of the source, i.e., $r_{l}^{2}=$ $h^{2}+(d-a)^{2}$ and $r_{2}^{2}=h^{2}+(d+a)^{2}$. The angle $\psi_{0}(t)$ is half the arc angle of the $\Gamma$-curve covering the source region (Fig. 5). It is given by

$$
\psi_{0}(t)=\cos ^{-1}\left[\frac{c^{2} t^{2}+d^{2}-a^{2}-h^{2}}{2 d \sqrt{c^{2} t^{2}-h^{2}}}\right]
$$

where $d^{2}=|\vec{x}|^{2}-h^{2}$ (i.e., $d$ is the distance of the observer from the $x_{3}$-axis). In the range $\frac{r_{1}}{c}<t<\frac{r_{2}}{c}$, Eq. (27) gives:

$$
\pi p^{\prime}(\vec{x}, t)=\frac{h I \psi_{o}^{\prime}(t)}{c t}
$$

From Eq. (28), we obtain

$$
\begin{aligned}
& \psi_{0}^{\prime}(t)= \\
& \frac{c^{2} t\left(|\vec{x}|^{2}-a^{2}-c^{2} t^{2}\right)}{\left(c^{2} t^{2}-h^{2}\right) \sqrt{4 d^{2}\left(c^{2} t^{2}-h^{2}\right)-\left(c^{2} t^{2}+d^{2}-a^{2}-h^{2}\right)^{2}}}
\end{aligned}
$$

Substitution of this result into Eq. (29) gives the acoustic pressure in this range as required. However, $\psi_{0}^{\prime}(t)$ is singular as $t \rightarrow \frac{r_{1}}{c}$ or $t \rightarrow \frac{r_{2}}{c}$. This can be tested eaily by substituting $c^{2} t^{2}=$ $r_{l}^{2}={ }^{c} h^{2}+\left(d^{c}-a\right)^{2}$ and $c^{2} t^{2}=h^{2}+(d+a)^{2}$ into Eq. (29). Therefore, in region 1 (i.e., outside the cylinder of radius $a$ ) we get an acoustic signal with infinite pressure in its front and tail. Note, however, that this acoustic pressure is integrable in time so that a microphone will give a finite, but large, signal.

When the observer is region 2 (i.e., inside the cylinder of radius $a$ ) then $d<a$ and we obtain from Eq. (26) the same result as Eq. (27), except that there is now an impulsive pressure at exactly the time $t=\frac{h}{c}$ of the magnitude $I$. The equation of this signal can be written as:

$$
p^{\prime}(\vec{x}, t)=I \delta\left(t-\frac{h}{c}\right)
$$

Initially, this impulsive pressure does not decay with distance from the surface and is confined to the cylindrical region of radius $a$ with axis along $x_{3}$-axis (i.e., a highly directional signal). Again using Eq. (29), we can show that we have other (integrable) singularities at $t=\frac{r_{1}}{c}$ and $t=\frac{r_{2}}{c}$ in the acoustic pressure $p^{\prime}(\vec{x}, t)$.

These results have serious implications for blade-vortex interaction noise studies. First, there is the possibility of highly directional impulsive sound which does not initially decay as $\frac{1}{r}$ from the blade surface. Such a phenomenon is observed in some experiments, Ref. 27 . Second, there is the possibility of large acoustic pressure amplitude from blade-vortex interaction in linear acoustic calculations. For this reason, it is not a good idea to compare peak to peak values of measured acoustic pressure signatures with predictions based on Eq. (5). The reasons are that:

i) The current acoustic codes are not designed to capture impulsive surface pressure of the type observed. They use formulations and algorithms not suitable for calculation of this kind of noise in blade-vortex interaction.

ii) The microphone has integrated the signal over its surface so that the peak values have been reduced.

The remedies for the above problems are:

i) The acoustic codes should be modified to use two time scales for calculations, one for the conventional thickness and loading calculations and a second with much finer time steps for blade-vortex interaction noise calculation.

ii) The code should be able to detect impulsive blade surface pressure. Careful analytical study, of the type presented in the above example, is needed to select a good formulation suitable for calculation of the sharp pulses generated by impulsive surface pressure. Equation (5) is perhaps a poor choice. A recent publication by Joshi, Liu and Boxwell, Ref. 28, evaluates codes based on two different formulations of Farassat, Refs. 15 and 18.

iii) Peak to peak comparison of experimental data with predictions is not meaningful if the predictions do not take into account the finite area of the microphone. A better comparison is based on the acoustic spectrum. In this connection, it must be mentioned that an understanding of the microphone and the associated equipment response to impulsive acoustic pressure is needed in interpretation of the measured acoustic data.

\section{Concluding Remarks}

In this paper, we have discussed many improvements in the application of the acoustic analogy to rotor noise prediction. In particular, we have shown how the noise from the shock surfaces and the quadrupoles in the boundary layer can be calculated. These calculations can all be made similar to the surface integrals of the thickness and loading noise. Additionally, tip vortex and blade wakes contribute quadrupole noise which can again be calculated. Order of magnitude analysis shows that the shock surfaces and boundary layer quadrupoles should be included in acoustic calculations, and both have directivity similar to the thickness noise.

We must emphasize the importance of theoretical acoustic analysis in rotor noise research. The acoustic analogy is a powerful method with great potential and capable of prediction of rotor noise. This potential is not realized if careful analysis of formulations and algorithms used in development of codes are not performed. Confusion has arisen in the past when this procedure was not followed and when sweeping statements about the unsuitability of linear analysis for rotor noise prediction have been made.

Finally, the acoustic analogy would be useless if good aerodynamic data were not available. There are codes under development which will eventually supply the unsteady blade surface pressure. However, it is imperative that experimental flow surveys be performed near the blade for both theoretical aerodynamicists and rotor acousticians.

\section{References}

'Ffowcs Williams, J. E., "The Acoustic Analogy-Thirty Years On," IMA Journal of Applied Mathematics, Vol. 32, 1984. 
${ }^{2}$ Ffowcs Williams, J. E., and Hawkings, D. L., "Sound Generated by Turbulence and Surfaces in Arbitrary Motion," Phil. Trans. Roy. Soc., London, A264, 1969.

${ }^{3}$ Farassat, F., "Linear Acoustic Formulas for Calculation of Rotating Blade Noise," AIAA Journal, 19, (9), 1981.

${ }^{4}$ Hanson, D. B., "Compressible Helicoidal Surface Theory for Propeller Aerodynamics and Noise," AIAA Journal, 21, (6), June 1983.

${ }^{5}$ Farassat, F., and Myers, M. K., "Aerodynamics via Acoustics: Application of Acoustic Formulas for Aerodynamic Calculations," AIAA paper 86-1877, July 1986.

'Hawkings, D. L., "Noise Generation by Transonic Open Rotors," in Mechanics of Sound Generation in Flows, E. A. Müller (ed.), Springer Verlag, Berlin, 1979.

${ }^{7}$ Korkan, K. D., Von Lavante, E., and Bober, L. J., “"Numerical Evaluation of Propeller Noise Including Nonlinear Effects," AIAA Journal, 24, 1986.

${ }^{8}$ Lyrintzis, A. S., and George, A. R., "Transonic Blade-Vortex Interactions: The Far Field,' Proceedings of the AHS National Specialists' Meeting on Aerodynamics and Aeroacoustics, Arlington, Texas, Feb. 25 27, 1987.

${ }^{9}$ Purcell, T. W., Strawn, R. C., and Yu, Y. H., "Prediction of HighSpeed Rotor Noise with a Kirchhoff Formula, Proceedings of the AHS National Specialists' Meeting on Aerodynamics and Aeroacoustics, Arlington, Texas, Feb. 25-27, 1987.

${ }^{10}$ Farassat, F., and Myers, M. K., "Extension of Kirchhoff's Formula to Radiation from Moving Surfaces, NASA TM 89149, May 1987 (to appear also in Journal of Sound and Vibration).

"Morgans, W. R., "The Kirchhoff Formula Extended to a Moving Surface,"' Philosophical Magazine, 9 (S.7 No. 55), 1930.

${ }^{12}$ Baeder, J. D., McCroskey, W. J., and Srinivasan, G. R., Acoustic Propagation using Computational Fluid Dynamics, Presented at the 42nd Annual Forum of the AHS, June 1986.

${ }^{13}$ Farassat, F., "Quadrupole Source in Prediction of the Noise of Rotating Blades-A New Source Description," AIAA Paper 87-2675, AIAA 11th Aeroacoustics Conference, Sunnyvale, California, October 19-21, 1987.

${ }^{14}$ McConnell, A. J., Applications of Tensor Analysis, Dover Publications, 1957.
${ }^{15}$ Farassat, F., "Theory of Noise Generation from Moving Bodies with an Application to Helicopter Rotors,' NASA TR R-451, December 1975.

${ }^{16}$ Farassat, F., and Brown, T. J., "A New Capability for Predicting Helicopter Rotor and Propeller Noise Including the Effect of Forward Motion," NASA TM X-74037, 1977.

${ }^{17}$ Nystrom, P. A., and Farassat, F., "A Numerical Technique for Calculation of the Noise of High-Speed Propellers with Advanced Blade Geometry," NASA TP 1662, 1980.

${ }^{18}$ Farassat, F., and Succi, G. P., "The Prediction of Helicopter Discrete Frequency Noise," Vertica, 7(4), 1983.

${ }^{19}$ Farassat, F., "Theoretical Analysis of Linearized Acoustics and Aerodynamics of Advanced Supersonic Propellers,' AGARD-CP-366, (10), $1-15,1985$

${ }^{20}$ Brentner, K. S., " A Prediction of Helicopter Rotor Discrete Frequency Noise for Three Scale Models Using a New Acoustics Program," AIAA Paper 87-0252, 1987.

${ }^{21}$ Hanson, D. B., and Fink, M., "'The Importance of Quadrupole Sources in the Prediction of Transonic Tip Speed Propeller Noise," $J$. Sound and Vib., 62(1), 1979

${ }^{22}$ Schmitz, F. H., and Yu, Y. H., "'Theoretical Modeling of High Speed Helicopter Impulsive Noise," Journal of the AHS, 24(1), 1979.

${ }^{23}$ Farassat, F., The Sound from Rigid Bodies in Arbitrary Motion, $\mathrm{PhD}$ thesis, Cornell University, Jan. 1973.

${ }^{24}$ Kitaplioglu, C., and George, A. R., " A Study of the Far-Field Sound Due to Unsteady Shocks on Rotors," AIAA Paper 77-1360, 1977.

${ }^{25}$ Schmitz, F. H., and Yu, Y. H., "Transonic Rotor Noise-Theoretical and Experimental Comparisons," Vertica, Vol. 5, 1981.

${ }^{26}$ Nakamura, Y., "Prediction of Blade-Vortex Interaction Noise from Measured Blade Pressure, NASA TM 81320, 1981.

${ }^{27}$ Martin, R. M., and Splettstoesser, W. R., "Acoustic Results of the Blade-Vortex Interaction Acoustic Test of a 40 Percent Model Rotor in the DNW," Proceedings of the AHS National Specialists' Meeting on Aerodynamics and Aeroacoustics, Arlington, Texas, Feb. 25-27, 1987.

${ }^{28}$ Joshi, M. C., Liu, S. R., and Boxwell, D. A., "Prediction of BladeVortex Interaction Using Measured Blade Pressures,' AIAA Paper 872749, AIAA 11th Aeroacoustics Conference, Sunnyvale, California, October $19-21,1987$ 